\title{
« La socialisation secondaire ne s'exerce pas sur une page blanche mais sur une page déjà écrite et déjà froissée par les expériences antérieures »
}

\author{
Entretien avec Muriel Darmon
}

\begin{abstract}
Muriel Darmon ${ }^{1}$, David Pichonnaz ${ }^{2}$, Kevin Toffel ${ }^{3}$
Muriel Darmon est sociologue et directrice de recherche au CNRS, dans le Centre européen de sociologie et de science politique (CNRS, École des hautes études en sciences sociales et Université Paris 1), et présidente de l'Association française de sociologie. Ses travaux ont porté notamment sur les processus de socialisation dans différentes institutions (groupes commerciaux d'amaigrissement, hôpitaux, écoles). Elle a récemment publié la troisième édition du manuel La socialisation, parue en 2016 aux éditions Armand Colin, Becoming anorexic: a sociological study chez l'éditeur Routledge en 2017, ainsi que Classes préparatoires. La fabrique d'une jeunesse dominante, paru en 2013 à La Découverte.
\end{abstract}

Vous êtes l'auteure d'un manuel sur la socialisation, dont la troisième édition est parue en 2016. Diriez-vous que l'étude de la socialisation est un domaine de spécialisation en sociologie?

MD : L'étude de la socialisation consiste à mener l'analyse sociologique du monde non pas à partir d'un domaine circonscrit de faits qui nous intéresserait en premier lieu, comme le travail ou l'école, mais à regarder un processus, la socialisation, qui se produit « partout ». L'étudier consiste à examiner la construction de dispositions individuelles, c'est-à-dire à regarder comment on apprend à agir de telle ou telle façon, à concevoir les choses d'une certaine manière et pas d'une autre. Il s'agit donc d'un regard qu'on peut appliquer à divers types d'objets et non d'une sociologie qu'on pourrait dire « sectorisée ». Je ne remets pas du tout en cause l'existence de sociologies thématiques, comme la sociologie du travail, ni les connaissances et les compétences particulières qui y sont développées : elles restent indispensables, et la sociologie de la socialisation

\footnotetext{
${ }^{1}$ Centre européen de sociologie et de science politique (CNRS, École des hautes études en sciences sociales et Université Paris 1).

${ }^{2}$ Haute école de travail social (HES-SO Valais-Wallis).

${ }^{3}$ Haute école de santé Vaud (HES-SO Haute école spécialisée de Suisse occidentale).
} 
n'a pas à se substituer aux approches spécialisées. Mais je fais le pari que ce regard peut apporter quelque chose, potentiellement, à tous les objets, le travail comme d'autres. Même en n'étant pas spécialistes d'une thématique, les sociologues de la socialisation peuvent apporter, sur un thème donné, leurs questions et une démarche de recherche, ainsi qu'une attention particulière à certaines choses qui ne sont pas toujours vues, ou pas toujours vues comme intéressantes. Nous allons traquer les déterminations sociales que sont les apprentissages et leurs contextes, le passé social des individus et les influences présentes qui s'exercent sur eux. De ce point de vue, la sociologie de la socialisation est en fait intrinsèquement déterministe, sans que ce déterminisme ne soit total ni surtout mécanique.

C'est aussi l'idée qu'on peut aborder de cette manière potentiellement tous les objets, même s'ils sont très spécifiques, dans des domaines très spécialisés, en posant des questions générales, celles qui sont au fondement de la sociologie. Ces questions sont assez fondamentales : comment les individus sont-ils faits ? Pourquoi adoptent-ils telle pratique et pas telle autre, telle façon de penser et pas telle autre ? Il s'agit de questions auxquelles on doit donner des réponses issues d'études de terrain, sans remettre en question le caractère empirique de la sociologie et la nécessité de fonder la production de connaissance dans les diverses formes d'enquêtes et de matériaux recueillis.

Les approches dispositionnelles ont peu inspiré les études du travail et des professions. Quelles en sont les raisons?

MD: Dans la sociologie américaine, il y a eu un grand dynamisme dans les années 19501960 autour de la socialisation professionnelle, qui s'illustre de façon frappante dans la «lutte d'orientations sociologiques » entre fonctionnalistes et interactionnistes sur la formation médicalé. Mais il faut souligner que ces travaux se sont intéressés à la socialisation par le biais de la formation, en faisant des études de médecine le «prototype » des socialisations professionnelles. Sont donc d'autant plus précieuses les études interactionnistes de l'époque qui ont observé les socialisations professionnelles plus informelles, «sur le tas », ou qui s'exercent dans des métiers moins prestigieux ou institutionnalisés. Toutefois, dans tous ces cas, la question des socialisations antérieures, et de l'articulation de ces nouveaux apprentissages aux anciens reste discrète, même si elle n'est pas ignorée. Pour le dire autrement, l'aspect proprement transformateur des socialisations professionnelles reste secondaire par rapport à leur dimension formatrice (les individus passent « juste » de profane à médecin, ou d'étudiants membres des fraternities à étudiants en médecine). L'importance de la « socialisation adulte » (adult socialization) se fait au détriment de la prise en compte des effets des socialisations primaires, comme s'il fallait choisir d'accorder du poids soit à la socialisation primaire, soit

\footnotetext{
${ }^{4}$ Cf. Darmon (2016 : 77-90).
} 
à la socialisation secondaire, dans une sorte de jeu à somme nulle des facteurs explicatifs des comportements - ce que « gagne » l'une, l'autre le perd.

En fait, le texte qui selon moi va opérer un tournant n'est ni fonctionnaliste ni interactionniste : c'est La construction sociale de la réalité de Berger et Luckmann, paru en 1966. Là, il y a vraiment une importance forte donnée à la socialisation primaire (la façon dont sont constitués les individus), et à la socialisation secondaire. On n'est plus dans le jeu à somme nulle mais bien dans la question analytique de l'articulation des effets des deux types de socialisation, ce qui se rapproche beaucoup des questions que l'on se pose aujourd'hui en sociologie des socialisations professionnelles. Cela dit, on ne peut pas dire qu'avec Berger et Luckmann on ait une analyse dispositionnelle des socialisations professionnelles. Ils font une sociologie de l'intériorisation du monde social plus que de son incorporation, des conceptions de soi, des façons de voir et de se voir, de la continuité biographique, dans laquelle la question des pratiques n'est pas centrale et qui repose sur d'autres types de questions telles que « comment la socialisation secondaire traite-t-elle avec un moi déjà formé et avec un monde déjà intériorisé ? ». Ce qui n'est pas tout à fait la même chose que de poser la question des transformations qu'induit le passage par un autre monde lorsqu'on arrive avec un patrimoine dispositionnel ou un habitus, de se demander ce qui se passe quand ces dispositions-là sont transformées ou non par l'influence d'un autre univers social, avec d'autres forces et d'autres types de déterminations sociales. Bref, saisir ce que la socialisation secondaire professionnelle fait à la socialisation primaire reste largement à faire.

\section{Qu'en est-il de la situation actuelle?}

MD: Il me semble que la rencontre entre la sociologie dispositionnaliste et la sociologie des groupes professionnels ne s'est pas encore vraiment faite. Lors d'un colloque d'hommage à Claude Dubar, en 2016, je m'étais étonnée qu'il y ait eu si peu d'échanges scientifiques réels entre le laboratoire Printemps et le Groupe de recherche sur la socialisation (GRS), ou entre Claude Dubar et Bernard Lahire ${ }^{5}$. On assiste en fait dans les années 1990 à un parallélisme des travaux : sociologie dispositionnaliste de la socialisation du côté de Bernard Lahire et du GRS, sociologie de la socialisation professionnelle du côté de Claude Dubar - de fait plutôt une sociologie des identités professionnelles si on regarde son manuel sur la socialisation. Un indicateur de cette segmentation est que les travaux de Lahire n’ont été intégrés qu'au compte-gouttes par Dubar dans les rééditions successives de son manuel ${ }^{6}$, par rapport à une sociologie de l'identité (F. de Singly par exemple) avec laquelle il dialogue davantage. Claude Dubar proposait donc une sociologie de la socialisation et des identités professionnelles, mais sans intégrer les travaux en train de se constituer et de s'institutionnaliser autour de l'école lyonnaise de la socialisation. Il faut aussi dire que, de son côté, Lahire n'a pas

${ }^{5}$ Cf. Darmon (2018).

${ }^{6}$ Cf. Dubar, Tripier, Boussard (2015 [1998]). 
beaucoup travaillé les socialisations secondaires en général, et les socialisations professionnelles en particulier. On observe donc une sorte de parallélisme entre deux sociologies qui ont finalement peu dialogué. Or, l'analyse dispositionnelle est en train de gagner en importance dans les études du travail et des professions, en témoigne ce numéro, mais aussi des journées d'études, un numéro de Terrains \& Travaux qui est en cours d'élaboration, des thèses de sociologie en cours, etc. Autant d'indices que les choses bougent.

\section{Comment aborder la socialisation professionnelle d'un point de vue dispositionnaliste?}

MD : Les processus de socialisation secondaire s'inscrivent dans l'histoire des individus. Ils s'exercent sur des dispositions déjà présentes et constituées pour éventuellement, et pas systématiquement et pas de façon certaine, transformer les dispositions présentes. La question des transformations individuelles (éventuelles) produites par le monde du travail devrait être, dans la perspective qui est la mienne, au cœur des réflexions sur la socialisation secondaire. En effet, la socialisation professionnelle, comme d'autres socialisations secondaires telles que les socialisations conjugales ou politiques, ne s'exerce pas sur rien. Il ne s'agit pas, comme pour la socialisation primaire, d'une formation des individus au sens le plus fort : la socialisation secondaire s'exerce sur des individus qui sont déjà dispositionnellement constitués, pas sur une page blanche mais sur une page déjà écrite et déjà froissée par les expériences antérieures - pour reprendre la métaphore deleuzienne et lahirienne du « pli » de l'individu.

Pour étudier la socialisation professionnelle, il convient donc de prendre en compte à la fois la force de la socialisation primaire et la possibilité transformatrice de la socialisation secondaire. Contrairement à une approche, d'inspiration interactionniste, qui se concentrerait sur l'examen des socialisations secondaires, y compris informelles (" sur le tas »), mais sans regarder l'impact de la socialisation primaire, il faut tenir compte du fait que, quand les individus arrivent dans le monde du travail, ils sont porteurs de dispositions qui sont déterminantes sur leurs pratiques et qui, comme elles sont incorporées, ne peuvent pas être détruites ou inhibées sans un travail ou un contexte particuliers. Mais cela suppose aussi d'aller au-delà d'une perspective qui serait essentiellement " primo-déterministe » de la socialisation, considérant que l'individu est, suite à la socialisation et à la constitution d'un habitus primaire, terminé, clos et intransformable. À un modèle rigide qui considérerait qu'il n'y a qu'une actualisation des dispositions issues de la socialisation primaire dans les mondes professionnels, où l'on ne ferait qu'observer l'ajustement préétabli des dispositions aux postes suite au travail de sélection d'habitus ajustés aux professions, on peut donc opposer l'action transformatrice des mondes professionnels sur les dispositions des individus. C'est une possibilité que prennent en compte Bourdieu et Passeron lorsqu'ils parlent, dans La reproduction (1970), de certaines institutions comme étant capables de transformer l'ha- 
bitus primaire. On peut très bien penser que le monde du travail et certaines professions ont cet effet-là, qu'ils détiennent une puissance transformatrice sur les dispositions préexistantes. Une telle posture, qui combine la prise en compte de l'influence institutionnelle tardive et la force des socialisations antérieures renvoie aussi à ce que Goffman dit de «l'institution totale », lorsque qu'il affirme que la « culture importée » des reclus est importante pour comprendre comment l'institution totale exerce son effet sur eux. Autrement dit, même dans le cas d'une institution totale qui a un pouvoir extrêmement homogénéisant et fort sur les individus, la culture importée du reclus, le fait qu'il arrive avec un certain rapport au monde, peut influencer l'effet de l'institution sur lui-même et opère des variations au sein du groupe des reclus. C'est ce que j'ai essayé de montrer dans certains de mes travaux, que ce soit sur les patientes anorexiques, les clients des groupes d'amaigrissement ou les élèves des classes préparatoires, en m'intéressant aux effets transformateurs des pratiques professionnelles sur les destinataires du travail, au travail sur autrui des professionnels et à ses effets - je dois reconnaître que j'ai en revanche très peu travaillé sur les professionnels eux-mêmes.

Parallèlement à l'articulation entre socialisation primaire et socialisation professionnelle, quelles autres pistes de recherche proposez-vous pour faire une sociologie dispositionnelle du travail?

MD : Une piste de recherche que je trouverais intéressante serait d'opérer la rencontre qui n’a pas eu lieu dans les années 1990, en partant des travaux de Bernard Lahire sur la socialisation familiale primaire et en les appliquant au champ de la socialisation professionnelle : il s'agirait de faire un « Tableaux de famille $»^{7}$ en sociologie de la socialisation professionnelle, c'est-à-dire de prendre des outils qui ont montré leurs vertus en analyse des socialisations primaires et de les appliquer aux socialisations professionnelles. Cela voudrait dire, par exemple, recenser la pluralité des influences qui s'exercent sur les individus dans le monde du travail, et considérer les modalités concrètes de la transmission de dispositions ou de traits de la culture professionnelle, en tout premier lieu le temps et les occasions de socialisation. On pourrait ainsi s'intéresser aux personnes avec qui les professionnels interagissent et qui sont donc susceptibles de leur transmettre des dispositions, aux capitaux dont disposent ces professionnels et à la transmission de ces derniers, ou encore à la manière dont les groupes professionnels contrôlent l'acquisition de dispositions par les nouveaux entrants, mais comment aussi elle leur échappe. Cela revient à se demander, toujours en s'inspirant des travaux de Lahire, comment les socialisations professionnelles peuvent être plurielles. À rebours d'une socialisation professionnelle qui serait considérée comme se faisant à partir d'une instance unifiée par une institution professionnelle, par les professionnels qui

\footnotetext{
${ }^{7}$ Lahire (2012 [1995]).
} 
sont en position de pouvoir ou d'initiateurs par rapport aux nouveaux entrants, il s'agirait de regarder les différentes instances de socialisation, comme les pairs du même rang, les supérieurs, mais aussi les différentes formations professionnelles, qu'elles soient formelles ou non, et les moments de sociabilité informels, par exemple à la cantine.

Un autre domaine qui pourrait être investigué davantage est celui du rapport entre formations professionnelles formelles et informelles, l'apprentissage du métier « sur le tas ». Dans mon ouvrage sur la socialisation, je distingue les formations professionnelles très institutionnalisées, les écoles, les stages, des formations informelles. Les deux sont créatrices de dispositions, mais il faudrait regarder la manière dont elles se conjuguent ou se contredisent, et s'interroger sur la façon dont, dans certaines professions, on apprend davantage par des instances informelles, ou en tous cas au fait qu'on pense que c'est le cas. J'ai été amenée dans mon livre, au fil des éditions successives, à introduire de plus en plus d'études sur les socialisations professionnelles informelles. En sociologie des professions, on pourrait dire en grossissant le trait qu'il y a eu beaucoup de travaux sur les formations formelles conduisant aux professions à statut, et beaucoup de travaux sur les formations informelles conduisant aux métiers moins reconnus (prostituées, strip-teaseuses et chauffeurs de taxis, pour reprendre une énumération célèbre). Cela correspond à une différence sociale bien sûr : il n'y a pas l'ENA des chauffeurs de taxi d'un côté, et de l'autre on n'apprécierait pas l'idée que les médecins suivent une formation "sur le tas », on préfère qu'ils passent plus de dix ans en milieu académique et en stages. Mais il serait sans doute pertinent de s'intéresser davantage à la manière dont les médecins et les hauts-fonctionnaires sont également façonnés, en tant que professionnels, « sur le tas » ou à la cantine (j’y reviens !), ou encore dans les associations et clubs auxquels ils participent, bref à travailler l'effet des socialisations informelles même quand elles sont déniées, et inversement l'effet des socialisations formelles et scolaires antérieures sur certaines professions dont on dit qu'elles ne s'apprennent pas, ou qu'elles s'apprennent sur le tas - je pense par exemple à l'idée, reprise parfois par les sciences politiques, selon laquelle la profession politique est un métier " qui ne s'apprend pas ». Tout en faisant attention à ne pas confondre formation et socialisation, qui sont deux choses différentes. La « formation» est un terme social, voire politique, qui recouvre des pratiques et des institutions qui se revendiquent comme telles. La « socialisation » renvoie à la manière dont les acteurs, les pratiques, les institutions façonnent les individus, et la formation n'est qu'une instance de socialisation dans la pluralité de celles qui y contribuent, et dont notre travail est de rendre compte.

\section{Bibliographie}

BeRger P. L., LuCKMAN T. (2012 [1966]), La construction sociale de la réalité, Paris, Armand Colin. 
BOURDIEU P., PASSERON J.-C. (1970), La reproduction. Éléments pour une théorie du système d'enseignement, Paris, Éditions de Minuit.

DARMON M. (2013), Classes préparatoires. La fabrique d'une jeunesse dominante, Paris, La Découverte.

DARMon M. (2016), La socialisation, Paris, Armand Colin («128»).

DARMON M. (2018), « Socialisation: petite histoire d'un manuel », Idées économiques et sociales, $\mathrm{n}^{\circ} 191$, p. 6-14.

DARMON M. et GARNIER L. (2017), Becoming anorexic: a sociological study, Londres, Routledge.

Dubar C., Tripier P., Boussard V. (2015 [1998]), Sociologie des professions, Paris Armand Colin.

LAHIRE, B. (2012 [1995]), Tableaux de familles. Heurs et malheurs scolaires en milieux populaires, Paris, Gallimard/Le Seuil (« Point »). 
